\title{
Intraprostatic injection of botulinum toxin type- A relieves bladder outlet obstruction in human and induces prostate apoptosis in dogs Yao-Chi Chuang1, Chieh-Hsien Tu², Chao-Cheng Huang ${ }^{3}$, Hsin-Ju Lin ${ }^{2}$, Po- Hui Chiang ${ }^{1}$, Naoki Yoshimura ${ }^{4}$ and Michael B Chancellor*4
}

\begin{abstract}
Address: ${ }^{1}$ Department of Urology, Chang Gung Memorial Hospital Kaohsiung Medical Center, Chang Gung University College of Medicine, Kaohsiung, Taiwan, ${ }^{2}$ Department of Veterinary Medicine, National Pingtung University of Science and Technology, Taiwan, ${ }^{3}$ Department of Pathology, Chang Gung Memorial Hospital Kaohsiung Medical Center, Chang Gung University College of Medicine, Kaohsiung, Taiwan and ${ }^{4}$ Department of Urology, University of Pittsburgh School of Medicine, Pittsburgh, Pennsylvania, USA

Email: Yao-Chi Chuang - chancellormb@Upmc.edu; Chieh-Hsien Tu - chancellormb@upmc.edu; ChaoCheng Huang - chancellormb@upmc.edu; Hsin-Ju Lin - chancellormb@upmc.edu; Po-Hui Chiang - chancellormb@upmc.edu; Naoki Yoshimura - chancellormb@upmc.edu; Michael B Chancellor* - chancellormb@upmc.edu

* Corresponding author
\end{abstract}

Published: 18 April 2006

BMC Urology 2006, 6:12 doi:10.1186/147I-2490-6-12

This article is available from: http://www.biomedcentral.com/I47I-2490/6/I2

(c) 2006 Chuang et al; licensee BioMed Central Ltd.

This is an Open Access article distributed under the terms of the Creative Commons Attribution License (http://creativecommons.org/licenses/by/2.0), which permits unrestricted use, distribution, and reproduction in any medium, provided the original work is properly cited.

\begin{abstract}
Background: With the increasing interest with botulinum toxin - A (BTX-A) application in the lower urinary tract, we investigated the BTX-A effects on the canine prostate and also in men with bladder outlet obstruction (BOO) due to benign prostatic hyperplasia (BPH).

Methods: Transperineal injection into the prostate using transrectal ultrasound (TRUS) was performed throughout the study. Saline with or without $100 \mathrm{U}$ of BTX-A was injected into mongrel dogs prostate. One or 3 months later, the prostate was harvested for morphologic and apoptotic study. In addition, eight BPH patients refractory to $\alpha$-blockers were treated with ultrasound guided intraprostatic injection of $200 \mathrm{U}$ of BTX-A.

Results: In the BTX-A treated dogs, atrophy and diffuse apoptosis was observed with H\&E stain and TUNEL stain at I and 3 months. Clinically, the mean prostate volume, symptom score, and quality of life index were significantly reduced by $18.8 \%, 73.1 \%$, and $61.5 \%$ respectively. Maximal flow rate significantly increased by $72.0 \%$.
\end{abstract}

Conclusion: Intraprostatic BTX-A injection induces prostate apotosis in dogs and relieves BOO in humans. It is therefore a promising alternative treatment for refractory $\mathrm{BOO}$ due to $\mathrm{BPH}$.

\section{Background}

Benign prostatic hyperplasia (BPH) is a common feature of the aging male and is regarded as a major cause of bladder outlet obstruction (BOO), which results in obstructive and irritative voiding symptoms. The bothersome urinary symptoms decrease the quality of life of these patients [1]. Transurethral resection of prostate (TURP) has been considered as a gold standard for the treatment of sympto- matic BPH. However, there have been concerns about the safety of TURP, with long-term morbidity including retrograde ejaculation, bladder neck contracture, and impotence [2-4]. Thus, there has been much interest in the development of alternative treatments. However, one feature of any of the minimally invasive techniques in clinical use to date is that they are permanent. Some men are 

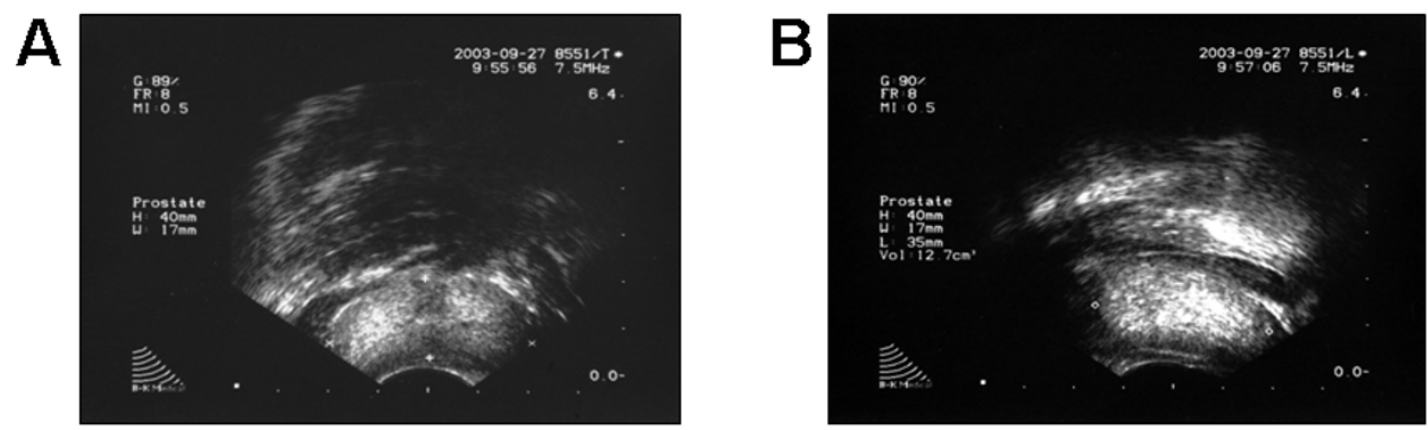

Figure I

Diffusion of hyperechoic BTX-A over the canine prostate immediate after BTX-A injection. A. Transverse view. B. Longitudinal view.

simply not ready or willing to undergo an irreversible procedure in order to treat their symptomatic BPH.

Botulinum toxin A (BTX-A) has been shown to block the release of neurotransmitters such as acetylcholine at the neuromuscular junction as well as in autonomic neurons [5-7]. BTX-A has been successfully used to treat various conditions including blepharospasm, strabismus, focal dystonias, and achalasia [7]. Injection of BTX-A into the urethra or bladder is an effective treatment for various types of lower urinary tract dysfunction for 6 months or longer [7-9]. Furthermore, previous studies in the rats have shown that intraurethral injection of BTX-A has significant inhibitory effects on urethral norepinephrine release [10] and intraprostatic injection of BTX-A induces selective denervation and subsequent atrophy of the glands [11]. Recent clinical studies have shown promising results after injection of BTX-A into lateral lobes of prostate for relieving voiding dysfunction due to $\mathrm{BPH}$ [12-14]. However, the histological change of prostatic tissue and mechanisms after BTX-A injection was not clear. The dog is one of a few animals that can develop BPH spontaneously and is frequently used as an animal model for human prostatic hyperplasia $[15,16]$. Therefore, the present study investigated morphologic and apoptotic change in the canine prostate after BTX-A injection. We also reported 8 cases of symptomatic $\mathrm{BPH}$ patients treated with BTX-A.

\section{Methods}

\section{Animal study}

The animal study was conducted at veterinary hospital, National Pingtung University, with the approval of the institutional review board. Eight mature male mongrel dogs, weighing 13 to $17 \mathrm{~kg}$., were used in this study. All animals were put on the lithotomy position and received perioperative antibiotics and intravenous sedation with pentobarbital. One hundred units of botulinum toxin A (BTX-A, Botox ${ }^{\circledR}$, Allergan, Irvine CA) were dissolved in 4 $\mathrm{ml}$ of $0.9 \%$ saline solution. Each lobe of the prostate received transperineal injection of $2 \mathrm{ml}$ of BTX-A with one-needle pass under TRUS guidance (Fig. 1) [16,17]. The control animals received the same procedures with injection of normal saline.

One or three months after BTX-A or normal saline injection ( $N=2$, in each group), the prostate was harvested for histological examination. The prostatic tissues were embedded in paraffin blocks after formalin fixation. Consecutive sections were taken for morphologic examination with hematoxylin and eosin stain and for study of apoptosis with TUNEL stain (terminal deoxynucleotidyl-mediated deoxyuridine triphosphate nick end labeling stain) [18] using a commercially available kit (Detection Kit POD, Boehringer Mannhein, Mannhein, Germany). Apoptotic nuclei were identified by detected fluorescence.

\section{Human study}

The effects on the human prostate and lower urinary tract symptoms were evaluated on 8 men with symptomatic $\mathrm{BPH}$ who had large prostate volume (mean $61.6 \mathrm{ml}$ ) and inadequate responses or intolerable side effects to $\alpha$ blockers (Table 1). The study was approved by the institutional review board of Chang Gung Memorial Hospital Kaohsiung and informed consent was obtained. Eight men, mean age $71.1 \pm 1.9$ years, were treated. All patients had a prostate volume of greater than $40 \mathrm{ml}$ and a peak urine flow rate less than $12 \mathrm{ml} / \mathrm{s}$. All men had a benign digital rectal prostate exam and did not suspect clinical malignancy. Patient 3 had PSA value at $9 \mathrm{ng} / \mathrm{ml}$ but rejected biopsy. Patient 4 and 5 had been experiencing chronic urinary retention for more than 1 month and 
Table I: Patient profiles and results after botulinum toxin-A treatment.

\begin{tabular}{|c|c|c|c|c|c|c|c|c|}
\hline Patient & Age (yr) & $\begin{array}{l}\text { Prostate } \\
\text { volume }(\mathrm{ml}) \\
\text { Pre/Post }\end{array}$ & $\begin{array}{l}\text { PSA (ng/ml) } \\
\text { Pre/Post }\end{array}$ & IPSS Pre/Post & $\begin{array}{l}\text { QOL index } \\
\text { Pre/Post }\end{array}$ & $\begin{array}{l}\text { Peak Flow } \\
\text { (ml/s) Pre/ } \\
\text { Post }\end{array}$ & $\begin{array}{l}\text { Residual urine } \\
\text { (ml) Pre/Post }\end{array}$ & $\begin{array}{l}\text { Follow-up } \\
\text { (month) }\end{array}$ \\
\hline I & 75 & $45.0 / 40.6$ & $4.1 / 1.6$ & $16 / 2$ & $3 / 1$ & $6 / 11$ & $73 / 20$ & 8 \\
\hline 2 & 60 & $54.3 / 40.7$ & 4.1/3.2 & $22 / 15$ & $3 / 2$ & $9.8 / 13$ & $138 / 20$ & 6 \\
\hline 3 & 77 & I I6.0/85.0 & $9.0 / 6.6$ & $18 / 13$ & $4 / 2$ & $12 / 14$ & $18 / 15$ & 6 \\
\hline 4 & 75 & $51.8 / 36.4$ & $-/ 20.7$ & $26 / 2$ & $5 / 1$ & $0 / 12$ & $500 / 24$ & 4 \\
\hline 5 & 73 & $76.2 / 61.4$ & $-/ 5.2 ; 4.4$ & $25 / 5$ & $5 / 2$ & $0 / 12$ & $500 / 40$ & 4 \\
\hline 6 & 70 & $46.3 / 39.3$ & $3.6 /-$ & $11 / 1$ & $3 / 1$ & $12 / 15$ & $72 / 17$ & 4 \\
\hline 7 & 72 & $61.8 / 54.9$ & $3.3 / 3.5$ & $19 / 2$ & $4 / 2$ & $11 / 14$ & $100 / 48$ & 3 \\
\hline 8 & 67 & $41.3 / 41.0$ & $2.0 / 2.4$ & $15 / 1$ & $4 / 1$ & $9 / 12$ & $20 / 12$ & 3 \\
\hline
\end{tabular}

IPSS- international prostate symptom score.

QOL- quality of life.

-: lack of data.

were treated with an indwelling Foley catheter. All patients received perioperative antibiotics with cefazolin $1 \mathrm{~g}$ (i.v.) and intravenous sedation with propofol $50 \mathrm{mg}$ in the lithotomy position. Transperineal injection was done under TRUS guidance (Fig. 2) [17]. As previous study described, each lobe of the prostate received $100 \mathrm{U}$ of BTX-A (Botox ${ }^{\circledR}$, Allergan) dissolved in $4 \mathrm{ml}$ of normal saline [13]. Two injections of equal volume $(2 \mathrm{ml})$ were given in each lobe. The total dose of BTX-A was $200 \mathrm{U}$ for each patient. Urethral catheter drainage was not performed postoperatively, except in two patients with chronic indwelling urethral catheters. These two men with urinary retention were able to urinate spontaneously 1 week post BTX-A injection. Patient 4 who was lack of preinjection PSA data and was found with high PSA after removal of Foley catheter received biopsy and proved adenocarcinoma 3 months after injection. He received brachytherapy and was followed-up at our out patient clinic.

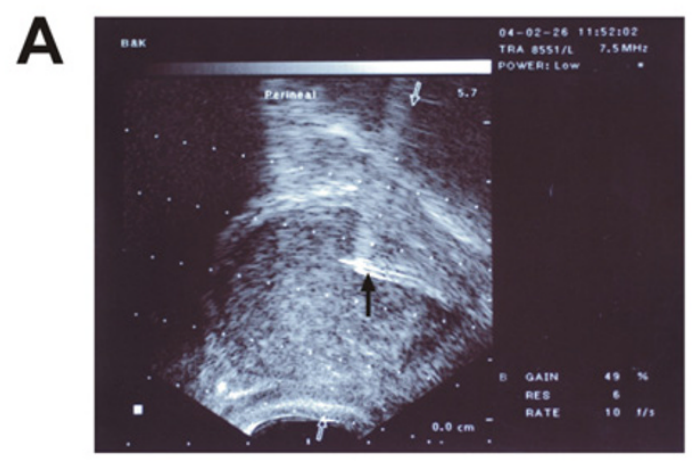

All men were evaluated pre and postoperatively for International Prostate Symptom Score (IPSS, 0-35, mild 0-7, moderate $8-18$, severe $\geq 19$ ), quality of life indices (QOL, $0-6$, indicating increasing severity of symptoms and low quality of life) [19], peak urinary flow rates, post-void residual volume detected by ultrasound, and prostate volume by TRUS $(0.52 \times$ Length $\times$ Height $\times$ Width $)$. Results were evaluated one month after the procedure. Quantitative data are expressed throughout this paper as means plus or minus standard error. Statistical analyses were performed using Student's t test for paired data with $\mathrm{p}<0.05$ considered significant.

\section{Results}

\section{Animal study}

None of the dogs suffered any complications including infection, incontinence, urinary retention, stones, and change in ambulation or weight loss. The average prostate volume was not significantly changed after saline or BTXA injection (from $11.8 \pm 0.9$ cc to $12.5 \pm 1.1 \mathrm{cc}$ and $11.0 \pm$

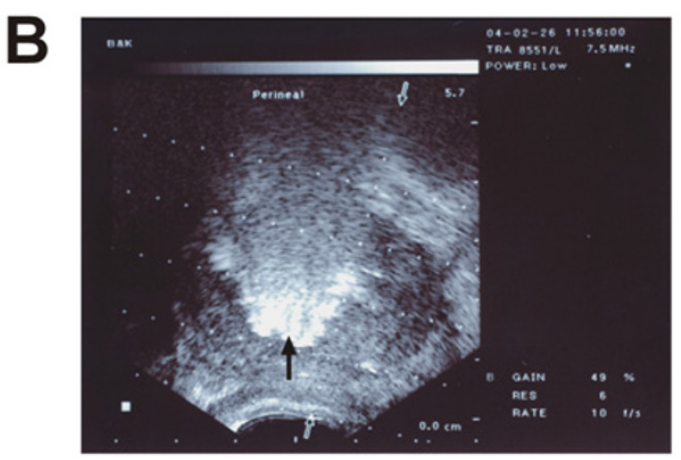

\section{Figure 2}

A. Confirmation of needle (black arrow) within the human prostate, longitudinal view. B. Diffusion of hyperechoic BTX-A (black arrow) over the prostate immediate post injection. 


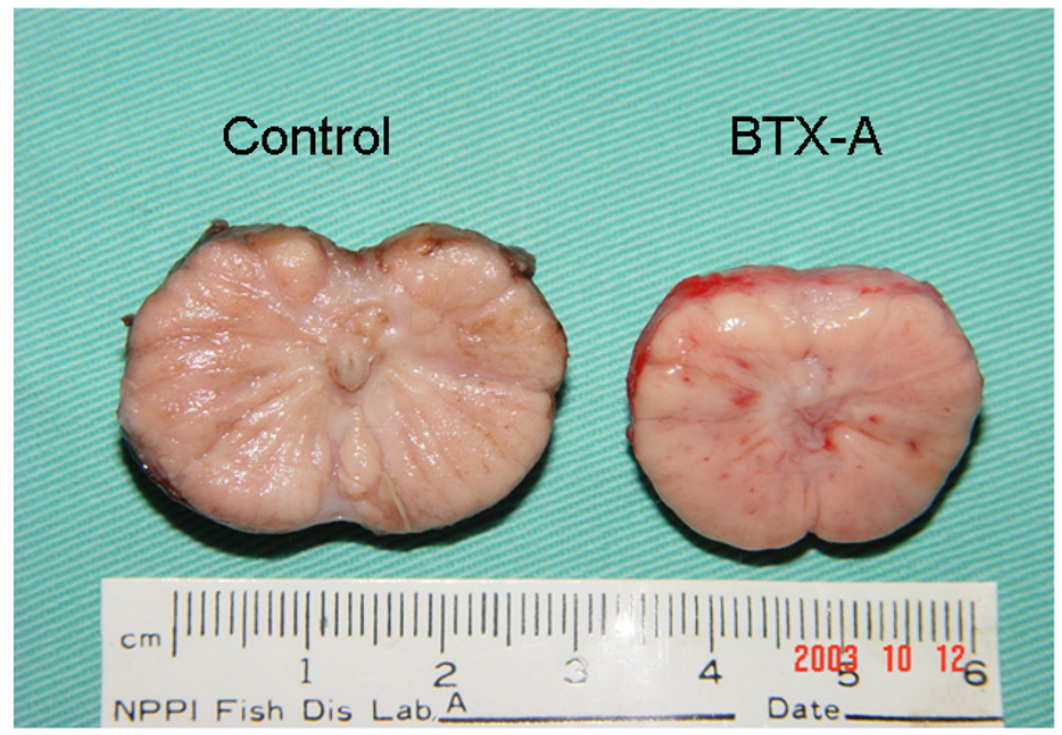

\section{Figure 3}

Coronal section of canine prostate one month after BTX-A or saline injection. Smaller in size and less indurations after BTX-A injection (B) than the control (A).

0.5 cc to $10.1 \pm 0.4 \mathrm{cc}$, respectively). However, gross examination of the prostate revealed smaller in size and less indurated after BTX-A injection than the control (Fig. 3). The H\&E stained sections of the prostate from the control animals of the 1 and 3 months groups revealed hypertrophied glands (Fig. 4A). However, the H\&E stained sections of the BTX-A treated animals from the 1 and 3 month post BTX-A treated groups revealed a degeneration of the prostate glands with similar results in both groups (Fig 4C).

The TUNEL staining of sections of control animals ( 1 and 3 month groups) revealed only a small amount of apoptosis (Fig. 4B). The TUNEL stained sections of tissue of BTX-A treated animals revealed fluorescence in the nuclei throughout the evaluated tissue, but fluorescence was predominantly seen in the nuclei of the glands (Fig. 4D). This significant glandular apoptosis was seen at both 1 and 3 month after intraprostatic BTX-A injection.

The proportion of TUNEL positive nuclei per 500 nuclei was quantified at $\times 400$ magnification using a $10 \times 10$ grid in the eyepiece in 4 fields. The mean apoptotic cells in the BTX-A treated group and control group were $187.5 \pm 20.4$ and $6.5 \pm 2.1$, respectively $(\mathrm{p}<0.05)$.

\section{Human study}

The follow-up period ranged from 3 to 8 months, mean $4.8 \pm 0.6$ months. No stress incontinence, retrograde ejaculation, erectile dysfunction, or systemic side effects were observed. Most patients reported improvement starting from 3 to 7 days post BTX-A treatment, reaching maximal effect in about 1 month, and maintaining effects as long as eight months.

At one month follow-up, the prostate volume, mean symptom score and quality of life index were significantly reduced by $18.8 \%$ (from $61.6 \pm 8.7$ to $50.0 \pm 5.9 \mathrm{ml}$, p < 0.05 ), $73.1 \%$ (from $19.0 \pm 1.8$ to $5.1 \pm 2.0, \mathrm{p}<0.05$ ) and $61.5 \%$ (from $3.9 \pm 0.3$ to $1.5 \pm 0.2, \mathrm{p}<0.05$ ) respectively. The maximal flow rate was increased by $72.0 \%$ (from 7.5 \pm 1.8 to $12.9 \pm 0.5 \mathrm{ml} / \mathrm{sec}, \mathrm{p}<0.05)$. The residual urine was decreased by $86.2 \%$ (from $177.6 \pm 71.7$ to $24.5 \pm 4.5$ $\mathrm{ml}, \mathrm{p}=0.064$ ). At three month follow-up, the effects were maintained and the prostate volume, mean symptom score, quality of life index, maximal flow rate, and residual urine were $49.5 \pm 5.9 \mathrm{ml}, 4.0 \pm 0.9,1.6 \pm 0.3,13.0 \pm$ $0.5 \mathrm{ml} / \mathrm{sec}$, and $22.1 \pm 3.0 \mathrm{ml}$, respectively.

\section{Discussion}

Although the etiology of BPH remains unknown, it has been suggested that $\mathrm{BPH}$ has two components: a static component that is related to prostatic enlargement and a dynamic component that reflects contraction of smooth muscle within the gland $[1,12]$. The static component is under parasympathetic control and regulated by androgen, while the stromal smooth muscle is sympathetically influenced [20,21]. Thus, drugs that relax smooth muscle within the prostate, such as $\alpha$-adrenergic receptor block- 

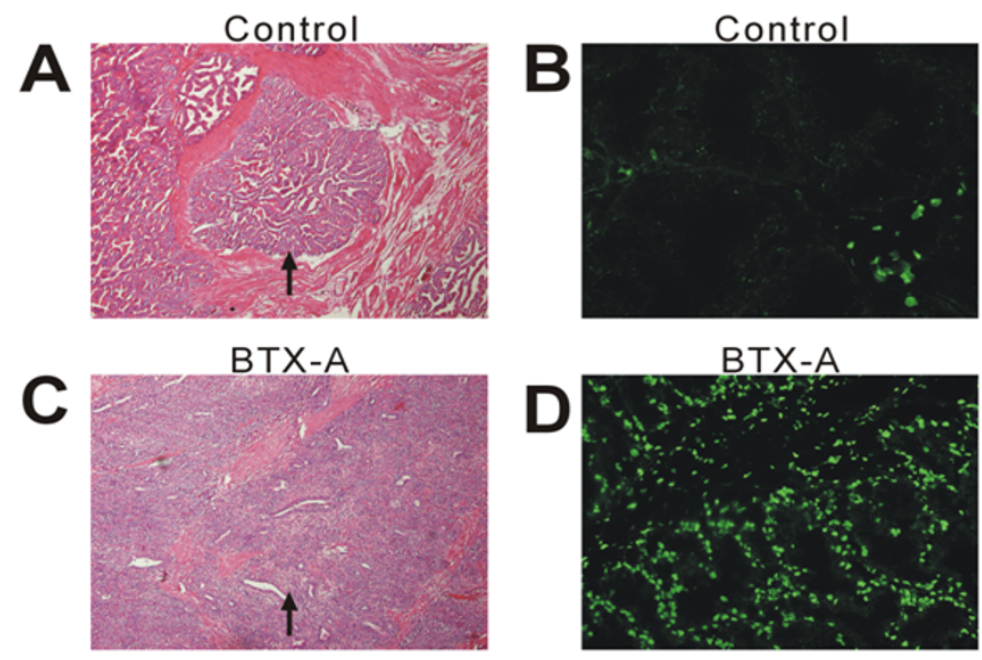

\section{Figure 4}

Photomicrographs and terminal deoxynucleotidyl-mediated deoxyuridine triphosphate nick end labeling (TUNEL) staining of prostate sections after saline (A,B) and BTX-A (C,D) injection at I month. Significant glandular proliferation with papillary infolding in the lumen was seen in the control canine $(A)$. Atrophy change of glandular component with flattening of the lining epithelium was seen in the BTX-A treated canine (C). Very few apoptotic nuclei are recognizable in the control animal (B). Strong fluorescence of apoptotic nuclei was seen in the BTX-A treated animal at I month. A, C magnification $\times 40$; B, D magnification $\times 100$.

ers, and drugs that shrink the volume, such as $5 \alpha$ reductase inhibitors, are used for the treatment of $\mathrm{BPH}$.

Recent evidence suggests that BPH could be originated from neural dysregulation of the prostate and alterations in local neuropeptides [20]. The prostatic neuroendocrine peptides may regulate the synthesis of prostatic secretory product, growth, and function [20,21]. Thus, BTX-A which has a cholinergic receptor predilection in its action on nerve terminals as well as inhibition of release of noradrenalin might regulate the neural control of prostate and relieve the symptoms of benign prostatic obstruction.

There are some differences between the canine and human prostate, nevertheless, experimental findings on the canine prostate can be regarded as valuable information. Previous studies have used the canine mode to elucidate the effects of orchiectomy and androgen on the prostate $[15,22]$. In the canine model, we used BTX-A 100 $\mathrm{U}$ for prostate injection, which dose might be about 2 times of the dose used in human (100 U for $15 \mathrm{~kg}$ in canine/200 U for $60 \mathrm{~kg}$ in human). However, the component of human prostate is different from the canine prostate. Species differences might have different responses to BTX-A treatment [23], therefore, the selected dose of BTXA $100 \mathrm{U}$ for the canine model cannot be completely interpreted to the human dose. Our present results suggest that injection of BTX-A into the canine prostate induced marked atrophy and diffuse apoptosis of prostate glands. The effect persisted for at least 3 months without any notable side effects. Doggweiler suggested that denervation can alter growth-factor expression in the prostate and resulted in programmed cell death [11]. Kyprianou demonstrated that the suppression of sympathetic tone on the prostate induced prostate apoptosis [24]. Expression of specific prostate apoptosis related genes, such as bcl 2 and transforming growth factor- $\beta$ has been implicated in the pathogenesis of BPH [24]. Thus, apoptotic changes in the prostate after BTX-A treatment are likely to be related with reduced neurotrophic influence on the gland. Therefore, the induction of apoptosis may emerge as an attractive target for the management of $\mathrm{BPH}$. As BPH might arise from neural dysregulation, BTX-A could potentially affect these neural mechanisms. Further study with staining of various neuropeptide markers in the dog prostate is undergoing for future report.

Although one of our patients did not reveal volume shrinkage, all of the 8 patients had improved in maximal flow rate, residual urine, IPSS score and quality of life indices, and had no side effects after BTX-A injection. The pathological features of BPH are heterogenous and include varying abnormalities of epithelium, smooth muscle and fibrous stroma. The relative proportion of epithelium and stroma is from $1: 2$ to $1: 5$ [24]. It is possible that the predominant component of the $\mathrm{BPH}$ nodule may 
determines the response to specific therapy, for example, smooth muscle predominant nodules would respond to $\alpha$-blockers and epithelial nodules to androgen-deprivation therapy $[1,24]$. The possibility of BTX-A effects on the dynamic component of BPH might explain the patient without volume shrinkage, but improved in lower urinary symptoms. Taken together, BTX-A might reduce the benign prostatic obstruction no matter what the detrusor contractility is.

The effects of denervation provided by the BTX-A will wear off, as new axons re-sprout in approximately 6 months [7]. Phelan et al reported that BTX-A effects on sphincter injection have prolonged subjective clinical efficacy beyond 6 months [8]. The beneficial results of the present study were evident within 1 month, and they continued throughout the follow-up period. The duration of BTX-A effects might depend on the different characteristic of targeted tissue, therapeutic dose, and intervals. Patients will determine if and when subsequent injections are necessary. Since the application of BTX-A on the treatment of lower urinary dysfunction is currently off-level use in Taiwan, it was necessary to be approved by the institutional review board for the human use and animal study. In support of evidence based medicine practices, caution should be applied until larger randomized clinical studies are completed that will guide physicians in making decisions about the use of botulinum toxin in the prostate.

\section{Conclusion}

In conclusion, our preliminary results suggest that BTX-A induces prostate atrophy and apoptosis in the canine and improves lower urinary tract symptoms in men with lower urinary tract symptoms due to benign prostatic hyperplasia. Intraprostate BTX-A injection may be a promising, reversible and alternative treatment for refractory $\mathrm{BPH}$.

\section{Competing interests}

The author(s) declare that they have no competing interests.

\section{Authors' contributions}

$\mathrm{YC} \& \mathrm{MB}$ composed the projects and write paper; $\mathrm{Y}$ and $\mathrm{PH}$ help to composed the project; CC do the histopathological review; $\mathrm{CH} \& \mathrm{HJ}$ do the animal study

\section{References}

I. Walsh PC: Treatment of benign prostatic hyperplasia. N Engl Med 1996, 335:557-563.

2. Roos NP, Wennberg JE, Malenka DJ, Fisher ES, McPherson K, Andersen TF, Cohen MM, Ramsey E: Mortality and reoperation after open and transurethral resection of the prostate for benign prostatic hyperplasia. N Engl J Med 1989, 320: I I 20- I I 24.

3. Bruskewitz RC, Larsen EH, Madsen P, Dorflinger T: 3-year followup of urinary symptoms after transurethral of the prostate. J Urol 1986, 136:613-615.

4. Roehrborn C, McConnell J, Barry M, Benaim E, Bruskewitz R, Blute $M$ : AUA guideline on management of benign prostatic hyper- plasia (2003). Chapter I: diagnosis and treatment recommendations. J Urol 2003, 170:530-547.

5. Jankovic J, Brin MF: Therapeutic uses of botulinum toxin. N Engl J Med 199I, 324: II86-II94.

6. MacKenzie I, Burnstock G, Dolly JO: The effects of purified botulinum neurotoxin type $A$ on cholinergic, adrenergic and nonadrenergic, atropine-resistant autonomi neuromuscular transmission. Neuroscience 1982, 7:997-1006.

7. Smith CP, Chancellor MB: Emerging Role of botulinum toxin in the treatment of voiding dysfunction. J Urol 2004, I71:2128-2137.

8. Phelan MW, Franks M, Somogyi GT, Yokoyama T, Fraser MO, Lavelle JP, Yoshimura N, Chancellor MB: Botulinum toxin urethral sphincter injection to restore bladder emptying in men and women with voiding dysfunction. J Urol 200I, 165: I I07-III0.

9. Kuo HC: Effect of botulinum a toxin in the treatment of voiding dysfunction due to detrusor underactivity. Urology 2003, 61:550-554.

10. Smith CP, Franks MF, McNeil BK, Ghosh R, deGroat WC, Chancellor $M B$, Somogyi GT: Effect of botulinum toxin $A$ on the autonomic nervous system of the rat lower urinary tract. J Urol 2003, 169:1896-1900.

II. Doggweiler R, Zermann DH, Ishigooka M, Schmidt RA: Botox induced prostatic involution. Prostate 1998, 37:44-50.

12. Maria G, Brisinda G, Civello IM, Bentivoglio AR, Sganga G, Albanese $A$ : Relief by botulinum toxin of voiding dysfunction due to benign prostatic hyperplasia: results of a randomized, placebo-controlled study. Urology 2003, 62:259-265.

13. Chuang YC, Chiang PH, Huang CC, Yoshimura N, Chancellor MB: Botulinum toxin type $\mathbf{A}$ improves benign prostatic hyperplasia symptoms in patients with small prostates. Urology 2005, 66:775-779.

14. Kuo HC: Prostate Botulinum toxin A injection- an alternative treatment for benign prostatic obstruction in poor surgical candidates. Urology 2005, 65:670-674.

15. Lin ATL, Chen MT, Chiang H, Yang CH, Chang LS: Effect of orchiectomy on the alpha adrenergic contractile response of dog prostate. J Urol 1995, 154:1930-1933.

16. Levy DA, Cromeens DM, Evans R, Stephens LC, von Eschenbach AC, Pisters LL: Transrectal ultrasound-guided intraprostatic injection of absolute ethanol with and without carmustine: a feasibility study in the canine model. Urology I999, 53:|245-I25I.

17. Chiang P-H, Chuang Y-C, Huang C-C, Chiang C-P: Pilot study of transperineal injection of dehydrated ethanol in the treatment of prostatic obstruction. Urology 2003, 61:797-80I.

18. Gavrieli $Y$, Sherman $Y$, Ben-Sasson SA: Identification of programmed cell death in situ via specific labeling of nuclear DNA fragmentation. I Cell Biol 1992, I I 9:493-50 I.

19. Cockett $A$, Aso Y, Denis L: Prostate symptom score and quality of life assessment. In Proceedings of the 2nd International Consultation on Benign Prostatic Hyperplasia (BPH) Paris Edited by: Cockett ATK, Khoury S, Aso Y, et al. Jersey, Channel Island, Scientific Communication International; 1994:553-555. June 27-30, 1993

20. Lepor $\mathrm{H}$, Baumann $M$, Shapiro $E$ : Identification and characterization of alpha I adrenergic receptors in the canine prostate using [ 1251]-Heat. J Urol 1987, I 38:1336-1339.

21. Lau WA, Pennefather JN, Mitchelson FJ: Cholinergic facilitation of neurotransmission to the smooth muscle of the guinea-pig prostate gland. British J Pharmacology 2000, 130:1013-1020.

22. Niu YJ, Ma TX, Zhang J, Xu Y, Han RF, Sun G: Androgen and prostatic stroma. Asian J Androl 2003, 5:19-26.

23. Chuang YC, Huang CC, Kang HY, Chiang PH, de Miguel F, Yoshimura $N$, Chancellor MB: Novel action of Botulinum toxin on the stromal and epithelial components of prostate gland. J Urol 2006, 173:1158-1163.

24. Kyprianou N: Doxazosin and terazosin suppress prostate growth by inducing apoptosis: clinical significance. J Urol 2003 , 169:1520-1525.

\section{Pre-publication history}

The pre-publication history for this paper can be accessed here:

http://www.biomedcentral.com/1471-2490/6/12/prepub 